Revista Destaques Acadêmicos, Lajeado, v. 12, n. 3, 2020. ISSN 2176-3070

DOI: http://dx.doi.org/10.22410/issn.2176-3070.v12i3a2020.2488

http://www.univates.br/revistas

\title{
HÁBITOS DE HIGIENE ORAL DE ESTUDANTES DE ESCOLA PÚBLICA DO INTERIOR DO CEARÁ
}

\author{
Cosmo Helder Ferreira da Silva ${ }^{1}$, Igor Oliveira de Queiroz ${ }^{2}$, \\ Francisco Gleuberson Oliveira Silva ${ }^{3}$, Guilherme Fernandes Gondim ${ }^{4}$, \\ Ana Caroline Rocha de Melo Leite ${ }^{5}$
}

Resumo: O trabalho educativo com pré-escolares e escolares deve ser priorizado, pois é nessa época que os indivíduos estão se descobrindo e aptos a aprender e adquirir bons hábitos de higiene oral e noções de conceitos em saúde bucal, o que vai refletir posteriormente em uma população mais consciente e informada a respeito da importância da prevenção, evitando tratamentos futuros. Objetivou-se conhecer os hábitos de higiene oral de escolares de 12 anos da escola Centro Educacional Dom Bosco da rede pública do município de Choró, Ceará. Trata-se de um estudo transversal, descritivo, quantitativo. Participaram do estudo 54 estudantes, com idade de 12 anos. Os resultados obtidos demonstram que, 59,3\% $(n=32)$ dos participantes eram do sexo feminino, sendo que $63 \%(n=34)$ escovam os dentes de 3 a 4 vezes ao dia e $57,4 \%(n=31)$ não fazem uso de fio dental, e $66,7 \%(n=36)$ muito satisfeito/satisfeito com sua condição de saúde bucal. Conclui-se que se se faz necessário conhecer as condições de saúde bucal de escolares, para traçar estratégias de promoção e prevenção da saúde com o objetivo de para melhorar os hábitos de saúde bucal e o acesso aos serviços odontológicos.

Palavras-chave: Higiene Oral; Saúde Bucal; Fatores Socioeconômicos.

1 Cirurgião-dentista, Docente do Curso de Odontologia do Centro Universitário Católica de Quixadá (UNICATÓLICA), Quixadá-Ceará, Pós-Graduado em Saúde da Família e Doutorando em Ciências da Saúde no Cento Universitário Saúde ABC (CUSABC), Santo André-São Paulo, E-mail: helderferreira_18@yahoo.com.br

2 Cirurgião-dentista, Centro Universitário Católica de Quixadá (UNICATÓLICA), QuixadáCeará, E-mail: igorchoro@hotmail.com

3 Acadêmico do Curso de Odontologia do Centro Universitário Católica de Quixadá, (UNICATÓLICA), E-mail: gleubersonoliveira1847@gmail.com

4 Acadêmico do Curso de Odontologia do Centro Universitário Católica de Quixadá, (UNICATÓLICA), E-mail: guiodonto2323@gmail.com

5 Cirurgiã-dentista, Docente do Curso de Enfermagem da Universidade da Integração Internacional da Lusofonia Afro-Brasileira (UNILAB), Doutora em Ciências Médicas pela Universidade Federal do Ceará (UFC), Fortaleza, Ceará, E-mail: acarolmelo@unilab.edu.br 


\section{INTRODUÇÃO}

A saúde bucal no Brasil apresenta níveis de precariedade que merecem uma atenção especial. Todavia, sabe-se que, para domínio e precaução de alguns problemas bucais, como a doença cárie, é importante aderir a procedimentos adequados de higiene bucal (OLIVEIRA et al., 2014).

No Brasil, a cárie dentária acomete $27 \%$ das crianças entre 18 e 36 meses de idade, este percentual chega a $59,4 \%$ aos 5 anos de idade. $70 \%$ de crianças aos 12 anos de idade possuem pelo menos um dente cariado (BRASIL, 2010). Vários fatores podem influenciar a saúde bucal, desde aspectos individuais ligadas ao consumo e estilo de vida até circunstâncias sociais, tais como fatores socioeconômicas e geográficas, acesso a recursos e serviços de saúde. Indivíduos com baixa renda possuem mais problemas e usam menos os serviços odontológicos pela própria dificuldade de acesso aos serviços públicos (GEUS et al., 2013).

É na infância que os bons hábitos de saúde bucal de cada indivíduo são primeiramente estabelecidos. $\mathrm{O}$ trabalho educativo com pré-escolares e escolares deve ser priorizado, pois é nessa época que os indivíduos estão se descobrindo e aptos a aprender e adquirir bons hábitos de higiene oral e noções de conceitos em saúde bucal, o que vai refletir posteriormente em uma população mais consciente e informada a respeito da importância da prevenção, evitando tratamentos futuros (PIVOTTO et al., 2013).

Nos dois últimos inquéritos epidemiológicos da população brasileira, realizados em 2002/2003 e 2010 ainda é evidente a necessidade de uma maior atenção às crianças de 12 anos, embora, os objetivos da Organização Mundial de Saúde (OMS) para o ano 2000, com índice de CPOD (Cariados, Perdidos, Obturados - Dentes) menor que 3,0 tenham sido alcançadas nesse grupo etário, escolhido pela OMS como a idade de monitoramento global da cárie dentária. Ressalta-se que para que haja o controle e a prevenção da cárie dentária, assim como outros problemas bucais, como a doença periodontal, é essencial a adesão a comportamentos adequados de higiene bucal, dentre outros relacionados à dieta e ao uso do flúor. Além disso, as crianças com 12 anos estão, em sua maioria, inseridas no ambiente escolar, sendo a escola um ambiente educacional e social propício para se trabalhar conhecimentos e mudanças de comportamento (OLIVEIRA et al., 2015).

A cárie dentária compõe o problema bucal que mais atinge crianças nas fases pré-escolar e escolar (CAVALCANTE; MATOS; CABRAL, 2014). Dentre os efeitos negativos da cárie sobre a vida das crianças destacam-se dificuldade de mastigar, diminuição do apetite, perda de peso, dificuldade para dormir, alteração no comportamento (irritabilidade e baixa autoestima) e diminuição do rendimento escolar (CASCAES,2014).

A escola reúne crianças em idades que favorecem a assimilação de medidas preventivas, como hábitos de higiene bucal, os quais são formados na 
infância. As atitudes e valores adquiridos entre os escolares nesta fase estarão presentes nas fases seguintes da vida (CAVALCANTE; MATOS; CABRAL, 2014). As medidas indicadas para a prevenção de lesões de cáries baseiam-se, basicamente, na educação e motivação do indivíduo ou população (FIGUEIRA; LEITE, 2008). O acesso à educação em saúde bucal é considerado um importante preditor para avaliação e melhoria das condições e dos comportamentos em saúde bucal (OLIVEIRA et al., 2014).

Portanto, este trabalho pode contribuir para as estratégias de avaliação e planejamento dos serviços odontológicos do município de Choró-CE com os escolares, para motivá-los da necessidade de uma higiene oral e educação da saúde bucal, com informações fundamentais para que as pessoas tenham o conhecimento sobre a saúde bucal, possibilitando uma melhor qualidade de vida.

Este estudo teve como objetivo conhecer os hábitos de higiene oral de escolares de 12 anos da escola Centro Educacional Municipal Dom Bosco da rede pública do município de Choró-CE.

\section{MÉTODO}

Este estudo foi aprovado pelo comitê de ética e pesquisa do Centro Universitário Católica de Quixadá, sob o número do parecer 3.175.362. Cada participante foi devidamente esclarecido sobre o estudo que foi realizado com a sua participação por meio da assinatura do Termo de Consentimento Livre e Esclarecido e Termo de Assentimento do Menor. A instituição onde foi realizado o estudo assinou a carta de anuência a fim de permitir a realização desse estudo.

Foi realizado um estudo transversal de natureza descritiva, quantitativa e qualitativa, com adolescentes do Centro Educacional Municipal Dom Bosco, do município de Choró-CE, localizado a aproximadamente $178 \mathrm{~km}$ da Capital do Ceará, Fortaleza.

A amostra foi do tipo não-probabilística por conveniência. Foram incluídos no estudo 54 alunos de 12 anos matriculados no Centro Educacional Municipal Dom Bosco. Foram excluídos do estudo os alunos que não responderam o questionário completo e que não estavam no dia e na hora da coleta de dados.

Para a coleta de dados foram aplicados um questionário estruturado adaptado de Silva et al., (2018a) e Silva et al., (2018b) com questões sobre hábitos de higiene bucal, dados socioeconômicos e o grau de satisfação em relação a saúde bucal dos participantes.

Os dados obtidos foram analisados de forma descritiva, categorizados e dicotomizados através da utilização do programa Microsoft Excel 2016 e em seguida foram submetidos à análise estatística por meio do Programa SPSS 20 para cruzamento das variáveis dependentes e independentes. 


\section{RESULTADOS}

Participaram do presente estudo 54 alunos com idade de 12 anos. Dentre as características sociodemográficos foi observado que grande parte dos alunos eram do sexo feminino $59,3 \%(n=32)$ com renda familiar menor ou igual a 1 salário-mínimo 79,6 $(\mathrm{n}=43)$. Dos participantes 70,4\% $(\mathrm{n}=38)$ residiam em zona urbana. No que se refere-se à frequência da escovação dos dentes $63 \%(n=34)$ escovam os dentes de 3-4 vezes ao dia. Em relação ao uso do fio dental 57,4\% $(n=31)$ registram não usarem e 40,7\% $(n=22)$ não fazem o uso de enxaguante bucal. Sobre a limpeza da língua $13 \%(n=7)$ não realizam a limpeza da língua durante a escovação. Sobre a ida ao dentista $24,1 \%(n=13)$ foram ao dentista há mais de 1 ano utilizando o serviço odontológico privado $24,1 \%(\mathrm{n}=13)$. Em relação há autopercepção $66,7 \%(n=36)$ apresenta-se satisfeito/muito satisfeito e 31,5\% (n=17) apresenta-se nem satisfeito/nem insatisfeito com sua condição de saúde bucal.

Tabela 1. Perfil sociodemográfico, autopercepção e hábitos de higiene oral de estudantes (n=54) da Escola Dom Bosco no município de Choró-CE, 2019.

\begin{tabular}{|c|c|c|}
\hline VARIÁVEIS & $\mathbf{N}$ & $\%$ \\
\hline \multicolumn{3}{|l|}{ Sexo } \\
\hline Masculino & 22 & 40,7 \\
\hline Feminino & 32 & 59,3 \\
\hline \multicolumn{3}{|l|}{ Renda familiar (em reais) } \\
\hline$\leq 1$ salário mínimo & 43 & 79,6 \\
\hline 1 a 2 salários mínimos & 11 & 20,4 \\
\hline \multicolumn{3}{|l|}{ Moradia } \\
\hline Zona urbana & 38 & 70,4 \\
\hline Zona rural & 16 & 29,6 \\
\hline \multicolumn{3}{|l|}{ Escovação dos Dentes } \\
\hline $1-2$ vezes & 20 & 37,0 \\
\hline 3-4 vezes & 34 & 63,0 \\
\hline \multicolumn{3}{|l|}{ Usa Fio Dental } \\
\hline Sim & 23 & 42,6 \\
\hline Não & 31 & 57,4 \\
\hline \multicolumn{3}{|l|}{ Enxaguante Bucal } \\
\hline Sim & 32 & 59,3 \\
\hline Não & 22 & 40,7 \\
\hline
\end{tabular}




\begin{tabular}{l|c|c}
\hline \multicolumn{1}{c|}{ VARIÁVEIS } & $\mathbf{N}$ & $\mathbf{\%}$ \\
\hline Limpeza da Língua & & \\
Sim & 47 & 87,0 \\
Não & 07 & 13,0 \\
Última Visita ao Dentista & & \\
6 meses & 41 & 75,9 \\
1 ano & 13 & 24,1 \\
Tipo de serviço & & \\
Público & 41 & 75,9 \\
Privado & 13 & 24,1 \\
Autopercepção & $\mathbf{N}$ & $\mathbf{\%}$ \\
Muito satisfeito/satisfeito & 36 & 66,7 \\
Nem satisfeito/nem insatisfeito & 17 & 31,5 \\
Insatisfeito/muito insatisfeito & 1 & 1,9 \\
TOTAL & $\mathbf{5 4}$ & $\mathbf{1 0 0 , 0}$ \\
\hline
\end{tabular}

Fonte: Autores, 2019.

\section{DISCUSSÃO}

A amostra do estudo constitui-se de 54 crianças sendo $100 \%$ delas com idade igual a 12 anos. Segundo Oliveira et al. (2015), ressaltou-se que não foram identificados estudos prévios que buscaram avaliar o acesso a tais informações entre escolares de 12 anos e uma limitação da pesquisa é que a entrevista ocorre apenas entre os adolescentes que frequentavam a escola e que estavam presentes no dia da aplicação do questionário. Já Oliveira et al. (2014), observou que não foram encontrados estudos prévios na literatura consultada que avaliaram a prevalência do acesso a orientações de higiene bucal entre escolares de 12 anos de idade.

Dentre as características sociodemográficos foi observado que grande parte dos alunos eram do sexo feminino $59,3 \%(n=32)$ com renda familiar menor ou igual a 1 salário-mínimo 79,6 ( $n=43)$. Segundo Oliveira et al. (2015) as mulheres tendem a procurar mais os serviços de saúde, por serem mais preocupadas com este assunto e isto aumenta suas chances de diagnosticar doenças e contribui para ampliar o acesso a práticas de promoção, prevenção e tratamento, e os grupos de renda mais baixa procuram menos os serviços de saúde. Coelho et al. (2011) também mostrou a prevalência de mulheres chegando a contabilizar $62 \%$ dos participantes da pesquisa. Para Oliveira et al. (2014) acredita-se que escolares da rede pública apresentam piores condições socioeconômicas e, consequentemente, podem apresentar piores condições de saúde bucal. No estudo de Bucker et al. (2011), a distribuição da renda familiar 
categorizada mostrou que quase a metade da amostra estudada $(48,06 \%)$ estava na faixa de até 1 salário-mínimo.

Todas as crianças residem na cidade de Choró, sendo que 70,4\% $(n=38)$ são de zona urbana. Para Magalhães et al. (2015), é coerente que grande parte da procura seja natural dessa região e áreas mais próximas devido a facilidade de informação da elevada oferta e funcionamento de referência em cuidados na atenção de saúde bucal.

No que se refere-se à frequência da escovação dos dentes $63 \%(n=34)$ escovam os dentes de 3-4 vezes ao dia. Segundo Davoglio et al. (2019) entre as crianças estudadas, a frequência de escovação dentária mostrou-se alta, similar a outros estudos. Já Freire et al. (2007) relatou que a freqüência de escovação foi alta em todos os grupos, o predomínio da frequência de três vezes ou mais ao dia tem sido também relatado nos demais estudos brasileiros. Para Pivotto et al. (2013) no presente estudo, os relatou que a frequência de escovação dental de suas crianças é de três vezes ao dia, corroborando com o relatado em outros trabalhos

O hábito de não fazer o usar do fio dental é observado em $57,4 \%$ (n=31) do total da amostra. Conforme Davoglio et al. (2009) uso diário de fio dental, esse foi maior entre os adolescentes de inserção socioeconômica mais alta e entre os que utilizavam os serviços privados, pois os usuários de serviços privados têm maior probabilidade de receberem instruções de higiene bucal no consultório e de terem poder aquisitivo para adquirir fio dental, o que facilitaria a adoção desse hábito. Já Pivotto et al. (2013) o fio dental embora a importante para a higienização das faces proximais já seja bem conhecida e a sua divulgação tenha crescido nos últimos anos, sua prática não é comum entre a população brasileira, a exemplo dos resultados encontrados no presente estudo e em outros. Oliveira et al (2015) ressalta-se que hábitos adequados de higiene bucal, como o uso de fio-dental, é maior entre escolares usuários de serviços odontológicos privados. Contudo Kubo e Mialhe (2011) acreditam que além de estimular a população a utilizar o fio dental é necessário educar as pessoas a fazerem seu uso de forma correta, a fim de que seu emprego seja eficiente na redução do biofilme e prevenção das doenças cárie e periodontal. Para Rodrigues et al. (2014), ressalta em sua pesquisa que o uso do fio dental é um hábito vinculado as consultas odontológicas, enquanto a escovação não, sugerindo que enquanto o uso do fio dental depende de orientações para se estabelecer como uma prática diária, a escovação já está consolidada.

Em relação ao enxaguante bucal 40,7\% (n=22) não fazem o uso e sobre a limpeza da língua $13 \%(n=7)$ não realizam a limpeza da língua durante a escovação. Para Sousa (2016) pouco mais de 50,0\% dos indivíduos analisados neste trabalho utilizam enxaguatório bucal pelo menos uma vez ao dia, com os objetivos de complementar a escovação e combater o mau hálito. Para Brandt (2014) uma parte da amostra (22\%) não faz a higienização da língua durante o processo de higiene bucal, porém a limpeza da língua também contribui para 
a redução da placa bacteriana, o que ressalta sua importância, já que a placa bacteriana é fator causador de grande parte das doenças bucais.

Sobre a ida ao dentista $24,1 \%(\mathrm{n}=13)$ foram ao dentista há mais de 1 ano utilizando o serviço odontológico privado $24,1 \%(n=13)$. Para Oliveira et al. (2014) todas as variáveis pertencentes aos serviços de saúde/custos com a saúde mantiveram-se associadas ao acesso a orientações de higiene bucal, constatou-se ainda que o acesso as referidas informações foram maiores entre escolares que utilizaram os serviços odontológicos privado. Segundo Oliveira et al. (2015) o presente trabalho mostrou que estes adolescentes buscaram mais o consultório privado, ou seja, a população com maior renda e escolaridade procura mais frequentemente os consultórios privados.

Em relação há autopercepção $66,7 \% \quad(n=36)$ apresenta-se satisfeito/ muito satisfeito e $31,5 \%(n=17)$ apresenta-se nem satisfeito/nem insatisfeito com sua condição de saúde bucal. Para Silveira et al. (2015) em um estudo em Pelotas-RS constatou-se que os adolescentes com melhor autopercepção da saúde bucal consultaram mais do que aqueles com autopercepção negativa, e aqueles que perceberam algum problema odontológico utilizaram $40 \%$ a mais os serviços quando comparados com aqueles que não perceberam. Já Geus et al. (2013) mostrou que a autopercepção da condição bucal tem sido utilizada como indicador do comportamento dos indivíduos quanto à busca por tratamentos odontológicos.

\section{CONCLUSÃO}

Constatou-se a partir da análise dos dados obtidos dos questionários do presente estudo foi possível traçar o perfil dos alunos de 12 anos da escola municipal Dom Bosco de Choró-Ce. A grande maioria pertence ao sexo feminino, com renda menor ou igual 1 salário mínimo, residem na zona urbana de Choró, escovam os dentes de 3 -4 vezes ao dia, não usam fio dental, usam enxaguante bucal, realizam a limpeza da língua, foram ao dentista nos últimos 6 meses, usaram o serviço público e acham muito satisfeito com sua saúde bucal. Este estudo demonstra a necessidade dos alunos em conhecer melhor os hábitos de higiene bucal, motivando-lhes a uma higiene oral e educação em saúde de qualidade. Podendo contribuir para as estratégias de avaliação e planejamento dos serviços odontológicos do município de Choró-CE com os escolares de 12 anos.

\section{REFERÊNCIAS}

BARBOSA, Taís de Souza et al. Qualidade de vida e saúde bucal em crianças e adolescentes: aspectos conceituais e metodológicos. Physis revista de saúde coletiva, v. 20, n. 1, p. 283-300, 2010. 
BOING, Antonio Fernando et al. Social determinants of health and dental caries in Brazil: a systematic review of the literature between 1999 and 2010. Revista Brasileira de Epidemiologia, v. 17, n. suppl 2, p. 102-115, 2014.

BUENO, Roberto Eduardo et al. Determinantes sociais e saúde bucal de adultos nas capitais do Brasil. Revista Panamericana de Salud Pública, v. 36, n. 1, p. 17-23, 2014.

COELHO, Mânia de Quadros et al. Análise comparativa do perfil dos usuários e acesso aos serviços de diagnóstico bucal de duas universidades de Minas Gerais, Brasil. Rev Arq Odontol, v. 47, n. 3, p.153-161, 2011.

DA SILVA, Cosmo Helder Ferreira et al. Conhecimento de usuários da Estratégia Saúde da Família sobre o câncer de boca. Arquivos em Odontologia, Belo Horizonte, v. 54: n. e07, 2018.

DA SILVA, Cosmo Helder Ferreira et al. Saúde bucal: dos hábitos e conhecimento de higiene ao comportamento e acesso a serviços odontológicos de universitários brasileiros e estrangeiros. Revista da Faculdade de Odontologia-UPF, Passo Fundo, v. 23, n. 1, p. 17-23, jan./abr. 2018.

DAVOGLIO, Rosane Silvia et al. Fatores associados a hábitos de saúde bucal e utilização de serviços odontológicos entre adolescentes. Cadernos de Saúde Pública, v. 25, n. 3, p. 655-667, 2009.

DE SOUZAA, Thaíse Borges Britto; DIASB, Juarez Pereira. Perfil Epidemiológico da dengue no município de Itabuna (Ba), 2000-Jun. 2009. Revista Baiana de Saúde Pública, v. 34, n. 3, p. 665-681, 2010.

FIGUEIRA, Taís Rocha et al. Percepções, conhecimentos e práticas em saúde bucal de escolares. RGO, v. 56, n. 1, p. 27-32, 2008.

FREIRE, Maria do Carmo Matias et al. Condição de saúde bucal em escolares de 12 anos de escolas públicas e privadas de Goiânia, Brasil. Revista Panamericana de Salud Pública, v. 28, n. 2, p. 86-91, 2010.

FREIRE, Maria do Carmo Matias; SHEIHAM, Aubrey; BINO, Yedda Avelino. Hábitos de higiene bucal e fatores sociodemográficos em adolescentes. Revista Brasileira de Epidemiologia, v. 10, n. 4, p. 606-614, 2007.

GUETERRES, Évilin Costa et al. Educação em saúde no contexto escolar: estudo de revisão integrativa. Enfermería Global, v. 16, n. 2, p. 464-499, 2017.

KUBO, Fabíola Mayumi Miyauchi; MIALHE, Fábio Luiz. Fio dental: da dificuldade ao êxito na remoção do biofilme interproximal. Arq. Odontol, v. 47, n. 1, p.51-55, 2011.

MAGALHÃES, Bruno Gama et al. Avaliação da qualidade dos serviços prestados pelos Centros de Especialidades Odontológicas: visão dos usuários. Cad Saúde Coletiva, v.23, n. 1, p.76-85, 2015. 
OLIVEIRA, Max Moura de et al. Fatores associados à procura de serviços de saúde entre escolares brasileiros: uma análise da Pesquisa Nacional de Saúde do Escolar (PeNSE), 2012. Cadernos de Saúde Pública, v. 31, n. 8, p. 1603-1614, 2015.

OLIVEIRA, Rodrigo Caldeira Nunes et al. Acesso a informações sobre como evitar problemas bucais entre escolares da Rede Pública de Ensino. Ciência \& Saúde Coletiva, v. 20, n. 1, p. 85-94, 2015.

OLIVEIRA, Rodrigo Caldeira Nunes et al. Acesso a orientações de higiene bucal entre escolares da rede pública de ensino. Revista de Odontologia da UNESP, v. 43, n. 6, p. 414-420, 2014.

PIVOTTO, Adriano et al. Hábitos de higiene bucal e índice de higiene oral de escolares do ensino público. Revista Brasileira em Promoção da Saúde, v. 26, n. 4, p. 455-461, 2013.

RODRIGUES, Laíse Angélica Mendes et al. Uso de serviços odontológicos entre pré-escolares: estudo de base populacional. Cien Saude Coletiva, v. 19, p. 4247-4256, 2014.

TAJRA, Fábio Solon et al. Uso do cariogram ${ }^{\circledR}$ na avaliação do risco de cárie em crianças em um município brasileiro: estudo piloto. Revista Brasileira em Promoção da Saúde, v. 27, n. 1, p. 62-71, 2014.

TESCH, Flávia Cariús; OLIVEIRA, Branca Heloísa de; LEÃO, Anna. Mensuração do impacto dos problemas bucais sobre a qualidade de vida de crianças: aspectos conceituais e metodológicos. Cadernos de Saúde Pública, v. 23, n. 11 p. 2555-2564, 2007. 